\title{
Application of Reproducing Kernel Method for Solving Nonlinear Fredholm-Volterra Integrodifferential Equations
}

\author{
Omar Abu Arqub, ${ }^{1}$ Mohammed Al-Smadi, ${ }^{2}$ and Shaher Momani ${ }^{3}$ \\ ${ }^{1}$ Department of Mathematics, Al-Balqa Applied University, Salt 19117, Jordan \\ ${ }^{2}$ Department of Mathematics and Information Technology, Tafila Technical University, \\ Tafila 66110, Jordan \\ ${ }^{3}$ Department of Mathematics, University of Jordan, Amman 11942, Jordan \\ Correspondence should be addressed to Omar Abu Arqub, o.abuarqub@bau.edu.jo
}

Received 24 June 2012; Revised 24 July 2012; Accepted 24 July 2012

Academic Editor: Irena Lasiecka

Copyright (C) 2012 Omar Abu Arqub et al. This is an open access article distributed under the Creative Commons Attribution License, which permits unrestricted use, distribution, and reproduction in any medium, provided the original work is properly cited.

\begin{abstract}
This paper investigates the numerical solution of nonlinear Fredholm-Volterra integro-differential equations using reproducing kernel Hilbert space method. The solution $u(x)$ is represented in the form of series in the reproducing kernel space. In the mean time, the $n$-term approximate solution $u_{n}(x)$ is obtained and it is proved to converge to the exact solution $u(x)$. Furthermore, the proposed method has an advantage that it is possible to pick any point in the interval of integration and as well the approximate solution and its derivative will be applicable. Numerical examples are included to demonstrate the accuracy and applicability of the presented technique. The results reveal that the method is very effective and simple.
\end{abstract}

\section{Introduction}

In recent years, there has been a growing interest in the integrodifferential equations (IDEs) which are a combination of differential and Fredholm-Volterra integral equations. IDEs are often involved in the mathematical formulation of physical phenomena. IDEs can be encountered in various fields of science such as physics, biology, and engineering. These kinds of equations can also be found in numerous applications, such as biomechanics, electromagnetic, elasticity, electrodynamics, fluid dynamics, heat and mass transfer, and oscillation theory [1-4]. 
The purpose of this paper is to extend the application of the reproducing kernel Hilbert space (RKHS) method to solve the nonlinear Fredholm-Volterra IDE which is as follows:

$$
u^{\prime}(x)+f(x) N(x, u(x))+\int_{a}^{b} k_{1}(x, t) G_{1}(u(t)) d t+\int_{a}^{x} k_{2}(x, t) G_{2}(u(t)) d t=g(x), \quad a \leq x, t \leq b,
$$

subject to the initial condition

$$
u(a)=\alpha,
$$

where $a, b, \alpha$ are real finite constants, $u \in W_{2}^{2}[a, b]$ is an unknown function to be determined, $f, g \in W_{2}^{1}[a, b], k_{1}(x, t), k_{2}(x, t)$ are continuous functions on $[a, b] \times[a, b], G_{1}(w), G_{2}(y)$, $N(x, z)$ are continuous terms in $W_{2}^{1}[a, b]$ as $w=w(x), y=y(x), z=z(x) \in W_{2}^{2}[a, b], a \leq x \leq$ $b,-\infty<w, y, z<\infty$ and are depending on the problem discussed, and $W_{2}^{1}[a, b], W_{2}^{2}[a, b]$ are reproducing kernel spaces.

In general, nonlinear Fredholm-Volterra IDEs do not always have solutions which we can obtain using analytical methods. In fact, many of real physical phenomena encountered are almost impossible to solve by this technique. Due to this, some authors have proposed numerical methods to approximate the solutions of nonlinear Fredholm-Volterra IDEs. To mention a few, in [5] the authors have discussed the Taylor polynomial method for solving IDEs $(1.1)$ and (1.2) when $N(x, u(x))=1, G_{1}(u(t))=u(t)$, and $G_{2}(u(t))=[u(t)]^{q}$, where $q \in \mathbb{N}$. The triangular functions method has been applied to solve the same equations when $N(x, u(x))=u(x), G_{1}(u(t))=[u(t)]^{p}$, and $G_{2}(u(t))=[u(t)]^{q}$, where $p, q \in \mathbb{N}$ as described in [6]. Furthermore, the operational matrix with block-pulse functions method is carried out in [7] for the aforementioned IDEs in the case $N(x, u(x))=1, G_{1}(u(t))=[u(t)]^{p}$, and $G_{2}(u(t))=[u(t)]^{q}$, where $p, q \in \mathbb{N}$. Recently, the Hybrid Legendre polynomials and BlockPulse functions approach for solving IDEs (1.1) and (1.2) when $N(x, u(x))=1, G_{1}(u(t))=$ $[u(t)]^{p}$, and $G_{2}(u(t))=[u(t)]^{q}$, where $p, q \in \mathbb{N}$ are proposed in [8]. The numerical solvability of Fredholm and Volterra IDEs and other related equations can be found in [9-11] and references therein. However, none of previous studies propose a methodical way to solve these equations. Moreover, previous studies require more effort to achieve the results, they are not accurate, and usually they are developed for special types of IDEs (1.1) and (1.2).

Reproducing kernel theory has important application in numerical analysis, differential equations, integral equations, probability and statistics, and so on [12-14]. Recently, using the RKHS method, the authors in [15-29] have discussed singular linear two-point boundary value problems, singular nonlinear two-point periodic boundary value problems, nonlinear system of boundary value problems, initial value problems, singular integral equations, nonlinear partial differential equations, operator equations, and fourth-order IDEs.

The outline of the paper is as follows: several reproducing kernel spaces are described in Section 2. In Section 3, a linear operator, a complete normal orthogonal system, and some essential results are introduced. Also, a method for the existence of solutions for (1.1) and (1.2) based on reproducing kernel space is described. In Section 4, we give an iterative method to solve (1.1) and (1.2) numerically in RKHS. Various numerical examples are presented in Section 5. This paper ends in Section 6 with some concluding remarks. 


\section{Several Reproducing Kernel Spaces}

In this section, several reproducing kernels needed are constructed in order to solve (1.1) and (1.2) using RKHS method. Before the construction, we utilize the reproducing kernel concept. Throughout this paper $\mathbb{C}$ is the set of complex numbers, $L^{2}[a, b]=\left\{u \mid \int_{a}^{b} u^{2}(x) d x<\infty\right\}$, $l^{2}=\left\{A \mid \sum_{i=1}^{\infty}\left(A_{i}\right)^{2}<\infty\right\}$, and the superscript $(n)$ in $u^{(n)}(t)$ denotes the $n$-th derivative of $u(t)$.

Definition 2.1 (see [18]). Let $E$ be a nonempty abstract set. A function $K: E \times E \rightarrow \mathbb{C}$ is a reproducing kernel of the Hilbert space $H$ if

(1) for each $t \in E, K(\cdot, t) \in H$,

(2) for each $t \in E$ and $\varphi \in H,\langle\varphi(\cdot), K(\cdot, t)\rangle=\varphi(t)$.

The last condition is called "the reproducing property": the value of the function $\varphi$ at the point $t$ is reproducing by the inner product of $\varphi$ with $K(\cdot, t)$. A Hilbert space which possesses a reproducing kernel is called a RKHS [18].

Next, we first construct the space $W_{2}^{2}[a, b]$ in which every function satisfies the initial condition (1.2) and then utilize the space $W_{2}^{1}[a, b]$.

Definition 2.2 (see [30]). $W_{2}^{2}[a, b]=\left\{u: u, u^{\prime}\right.$ are absolutely continuous on $[a, b], u, u^{\prime}, u^{\prime \prime} \in$ $L^{2}[a, b]$, and $\left.u(a)=0\right\}$. The inner product and the norm in $W_{2}^{2}[a, b]$ are defined, respectively, by

$$
\langle u, v\rangle_{W_{2}^{2}}=u(a) v(a)+u^{\prime}(a) v^{\prime}(a)+\int_{a}^{b} u^{\prime \prime}(y) v^{\prime \prime}(y) d y
$$

and $\|u\|_{W_{2}^{2}}=\sqrt{\langle u, u\rangle_{W_{2}^{2}}}$, where $u, v \in W_{2}^{2}[a, b]$.

Definition 2.3 (see [23]). $W_{2}^{1}[a, b]=\left\{u: u\right.$ is absolutely continuous on $[a, b]$ and $u, u^{\prime} \in$ $\left.L^{2}[a, b]\right\}$. The inner product and the norm in $W_{2}^{1}[a, b]$ are defined, respectively, by $\langle u, v\rangle_{W_{2}^{1}}=$ $\int_{a}^{b} u(t) v(t)+u^{\prime}(t) v^{\prime}(t) d t$ and $\|u\|_{W_{2}^{1}}=\sqrt{\langle u, u\rangle_{W_{2}^{1}}}$, where $u, v \in W_{2}^{1}[a, b]$.

In [23], the authors have proved that the space $W_{2}^{1}[a, b]$ is a complete reproducing kernel space and its reproducing kernel function is given by

$$
T_{x}(y)=\frac{1}{2 \sinh (b-a)}[\cosh (x+y-b-a)+\cosh (|x-y|-b+a)] .
$$

From the definition of the reproducing kernel spaces $W_{2}^{1}[a, b]$ and $W_{2}^{2}[a, b]$, we get $W_{2}^{1}$ $[a, b] \supset W_{2}^{2}[a, b]$.

The Hilbert space $W_{2}^{2}[a, b]$ is called a reproducing kernel if for each fixed $x \in[a, b]$ and any $u(y) \in W_{2}^{2}[a, b]$, there exist $K(x, y) \in W_{2}^{2}[a, b]$ (simply $\left.K_{x}(y)\right)$ and $y \in[a, b]$ such that $\left\langle u(y), K_{x}(y)\right\rangle_{W_{2}^{2}}=u(x)$. The next theorem formulates the reproducing kernel function $K_{x}(y)$. 
Theorem 2.4. The Hilbert space $W_{2}^{2}[a, b]$ is a reproducing kernel and its reproducing kernel function $K_{x}(y)$ can be written as

$$
K_{x}(y)= \begin{cases}\sum_{i=1}^{4} p_{i}(x) y^{i-1}, & y \leq x \\ \sum_{i=1}^{4} q_{i}(x) y^{i-1}, & y>x\end{cases}
$$

where $p_{i}(x)$ and $q_{i}(x)$ are unknown coefficients of $K_{x}(y)$.

Proof. Through several integrations by parts for (2.1), we obtain $\left\langle u(y), K_{x}(y)\right\rangle_{W_{2}^{2}}=\sum_{i=0}^{1}$ $u^{(i)}(a)\left(K_{x}^{(i)}(a)+(-1)^{i} K_{x}^{(3-i)}(a)\right)+\sum_{i=0}^{1}(-1)^{1-i} u^{(i)}(b) K_{x}^{(3-i)}(b)+\int_{a}^{b} u(y) K_{x}^{(4)}(y) d y$. Since $K_{x}(y) \in$ $W_{2}^{2}[a, b]$, it follows that $K_{x}(a)=0$. Also, since $u \in W_{2}^{2}[a, b]$, one obtains $u(a)=0$. Thus, if $K_{x}^{(i)}(b)=0, i=2,3$, and $K_{x}^{\prime}(a)-K_{x}^{\prime \prime}(a)=0$, then $\left\langle u(y), K_{x}(y)\right\rangle_{W_{2}^{2}}=\int_{a}^{b} u(y) K_{x}^{(4)}(y) d y$. Now, for each $x \in[a, b]$, if $K_{x}(y)$ also satisfies $K_{x}^{(4)}(y)=\delta(x-y)$, where $\delta$ is the dirac-delta function, then $\left\langle u(y), K_{x}(y)\right\rangle_{W_{2}^{2}}=u(x)$. Obviously, $K_{x}(y)$ is the reproducing kernel function of the space $W_{2}^{2}[a, b]$.

Next, we give the expression of the reproducing kernel function $K_{x}(y)$. The characteristic equation of $K_{x}^{(4)}(y)=\delta(x-y)$ is $\lambda^{4}=0$, and their characteristic values are $\lambda=0$ with 4 multiple roots. So, let the kernel $K_{x}(y)$ be as defined in (2.3).

On the other hand, let $K_{x}(y)$ satisfy $K_{x}^{(m)}(x+0)=K_{x}^{(m)}(x-0), m=0,1,2$. Integrating $K_{x}^{(4)}(y)=\delta(x-y)$ from $x-\varepsilon$ to $x+\varepsilon$ with respect to $y$ and letting $\varepsilon \rightarrow 0$, we have the jump degree of $K_{x}^{(3)}(y)$ at $y=x$ given by $K_{x}^{(3)}(x-0)-K_{x}^{(3)}(x+0)=-1$. Through the last descriptions the unknown coefficients of (2.3) can be obtained. This completes the proof.

By using Mathematica 7.0 software package, the representation of the reproducing kernel function $K_{x}(y)$ is provided by

$$
K_{x}(y)= \begin{cases}\frac{1}{6}(y-a)\left(2 a^{2}-y^{2}+3 x(2+y)-a(6+3 x+y)\right), & y \leq x \\ \frac{1}{6}(x-a)\left(2 a^{2}-x^{2}+3 y(2+x)-a(6+x+3 y)\right), & y>x\end{cases}
$$

The following corollary summarizes some important properties of the reproducing kernel function $K_{x}(y)$.

Corollary 2.5. The reproducing kernel function $K_{x}(y)$ is symmetric, unique, and $K_{x}(x) \geq 0$ for any fixed $x \in[a, b]$.

Proof. By the reproducing property, we have $K_{x}(y)=\left\langle K_{x}(\xi), K_{y}(\xi)\right\rangle=\left\langle K_{y}(\xi), K_{x}(\xi)\right\rangle=$ $K_{y}(x)$ for each $x$ and $y$. Now, let $K_{x}^{1}(y)$ and $K_{x}^{2}(y)$ be all the reproducing kernels of the space $W_{2}^{2}[a, b]$; then $K_{x}^{1}(y)=\left\langle K_{x}^{1}(\xi), K_{y}^{2}(\xi)\right\rangle=\left\langle K_{y}^{2}(\xi), K_{x}^{1}(\xi)\right\rangle=K_{y}^{2}(x)=K_{x}^{2}(y)$. Finally, we note that $K_{x}(x)=\left\langle K_{x}(\xi), K_{x}(\xi)\right\rangle=\left\|K_{x}(\xi)\right\|^{2} \geq 0$. 


\section{Introduction to a Linear Operator and a Normal Orthogonal System in $W_{2}^{2}[a, b]$}

In this section, we construct an orthogonal function system of $W_{2}^{2}[a, b]$. Also, representation of the solution of (1.1) and (1.2) is given in the reproducing kernel space $W_{2}^{2}[a, b]$.

To do this, we define a differential operator $L: W_{2}^{2}[a, b] \rightarrow W_{2}^{1}[a, b]$ such that $L u(x)=u^{\prime}(x)$. After homogenization of the initial condition (1.2), IDEs (1.1) and (1.2) can be converted into the equivalent form as follows:

$$
\begin{gathered}
\operatorname{Lu}(x)=F(x, u(x), T u(x), S u(x)), \quad a \leq x \leq b, \\
u(a)=0,
\end{gathered}
$$

such that $F(x, u(x), T u(x), S u(x))=g(x)-f(x) N(x, u(x))-T u(x)-S u(x), T u(x)=\int_{a}^{b} k_{1}$ $(x, t) G_{1}(u(t)) d t$, and $S u(x)=\int_{a}^{x} k_{2}(x, t) G_{2}(u(t)) d t$, where $u(x) \in W_{2}^{2}[a, b]$ and $F(x, w, y, z) \in$ $W_{2}^{1}[a, b]$ for $x \in[a, b]$ and $w=w(x), y=y(x), z=z(x) \in W_{2}^{2}[a, b],-\infty<w, y, z<\infty$. It is easy to show that $L$ is a bounded linear operator from $W_{2}^{2}[a, b]$ to $W_{2}^{1}[a, b]$.

Now, we construct an orthogonal function system of $W_{2}^{2}[a, b]$. Let $\varphi_{i}(x)=T_{x_{i}}(x)$ and $\psi_{i}(x)=L^{*} \varphi_{i}(x)$, where $\left\{x_{i}\right\}_{i=1}^{\infty}$ is dense on $[a, b]$ and $L^{*}$ is the adjoint operator of $L$. From the properties of the reproducing kernel $T_{x}(y)$, we have $\left\langle u(x), \varphi_{i}(x)\right\rangle_{W_{2}^{1}}=\left\langle u(x), T_{x_{i}}(x)\right\rangle_{W_{2}^{1}}=$ $u\left(x_{i}\right)$ for every $u(x) \in W_{2}^{1}[a, b]$. In terms of the properties of $K_{x}(y)$, one obtains $\left\langle u(x), \psi_{i}(x)\right\rangle_{W_{2}^{2}}=\left\langle u(x), L^{*} \varphi_{i}(x)\right\rangle_{W_{2}^{2}}=\left\langle L u(x), \varphi_{i}(x)\right\rangle_{W_{2}^{1}}=L u\left(x_{i}\right), i=1,2, \cdots$.

It is easy to see that $\psi_{i}(x) \stackrel{2}{=} L^{*} \varphi_{i}(x)=\left\langle L^{*} \varphi_{i}(x), K_{x}(y)\right\rangle_{W_{2}^{2}}=\left\langle\varphi_{i}(x), L_{y} K_{x}(y)\right\rangle_{W_{2}^{1}}=$ $\left.L_{y} K_{x}(y)\right|_{y=x_{i}}$. Thus, $\psi_{i}(x)$ can be expressed in the form $\psi_{i}(x)=\left.L_{y} K_{x}(y)\right|_{y=x_{i}}$, where $L_{y}$ indicates that the operator $L$ applies to the function of $y$.

Theorem 3.1. For (3.1), if $\left\{x_{i}\right\}_{i=1}^{\infty}$ is dense on $[a, b]$, then $\left\{\psi_{i}(x)\right\}_{i=1}^{\infty}$ is the complete function system of the space $W_{2}^{2}[a, b]$.

Proof. Clearly, $\psi_{i}(x) \in W_{2}^{2}[a, b]$. For each fixed $u(x) \in W_{2}^{2}[a, b]$, let $\left\langle u(x), \psi_{i}(x)\right\rangle_{W_{2}^{2}}=0, i=$ $1,2, \ldots$, which means that $\left\langle u(x), \psi_{i}(x)\right\rangle_{W_{2}^{2}}=\left\langle u(x), L^{*} \varphi_{i}(x)\right\rangle_{W_{2}^{2}}=\left\langle L u(x), \varphi_{i}(x)\right\rangle_{W_{2}^{1}}=L u\left(x_{i}\right)=$ 0 . Note that $\left\{x_{i}\right\}_{i=1}^{\infty}$ is dense on $[a, b]$; therefore, $L u(x)=0$. It follows that $u(x)=0$ from the existence of $L^{-1}$. So, the proof of the theorem is complete.

The orthonormal function system $\left\{\bar{\psi}_{i}(x)\right\}_{i=1}^{\infty}$ of the space $W_{2}^{2}[a, b]$ can be derived from Gram-Schmidt orthogonalization process of $\left\{\psi_{i}(x)\right\}_{i=1}^{\infty}$ as follows:

$$
\bar{\psi}_{i}(x)=\sum_{k=1}^{i} \beta_{i k} \psi_{k}(x)
$$

where $\beta_{i k}$ are orthogonalization coefficients given as $\beta_{11}=1 /\left\|\psi_{1}\right\|, \beta_{i i}=1 / d_{i k}$, and $\beta_{i j}=$ $-\left(1 / d_{i k}\right) \sum_{k=j}^{i-1} c_{i k} \beta_{k j}$ for $j<i$ in which $d_{i k}=\sqrt{\left\|\psi_{i}\right\|^{2}-\sum_{k=1}^{i-1} c_{i k^{\prime}}^{2}} c_{i k}=\left\langle\psi_{i}, \bar{\psi}_{k}\right\rangle_{W_{2}^{2}}$, and $\left\{\psi_{i}(x)\right\}_{i=1}^{\infty}$ is the orthonormal system in the space $W_{2}^{2}[a, b]$. 
Theorem 3.2. For each $u(x) \in W_{2}^{2}[a, b]$, the series $\sum_{i=1}^{\infty}\left\langle u(x), \bar{\psi}_{i}(x)\right\rangle \bar{\psi}_{i}(x)$ is convergent in the norm of $W_{2}^{2}[a, b]$. On the other hand, if $\left\{x_{i}\right\}_{i=1}^{\infty}$ is dense on $[a, b]$ and $u(x)$ is the exact solution of (3.1), then

$$
u(x)=\sum_{i=1}^{\infty} \sum_{k=1}^{i} \beta_{i k} F\left(x_{k}, u\left(x_{k}\right), T u\left(x_{k}\right), S u\left(x_{k}\right)\right) \bar{\psi}_{i}(x) .
$$

Proof. Since $u(x) \in W_{2}^{2}[a, b], \sum_{i=1}^{\infty}\left\langle u(x), \bar{\psi}_{i}(x)\right\rangle \bar{\psi}_{i}(x)$ is the Fourier series expansion about normal orthogonal system $\left\{\bar{\psi}_{i}(x)\right\}_{i=1}^{\infty}$, and $W_{2}^{2}[a, b]$ is the Hilbert space, then the series $\sum_{i=1}^{\infty}$ $\left\langle u(x), \bar{\psi}_{i}(x)\right\rangle \bar{\psi}_{i}(x)$ is convergent in the sense of $\|\cdot\|_{W_{2}^{2}}$. On the other hand, using (3.2), we have

$$
\begin{aligned}
u(x) & =\sum_{i=1}^{\infty}\left\langle u(x), \bar{\psi}_{i}(x)\right\rangle_{W_{2}^{2}} \bar{\psi}_{i}(x)=\sum_{i=1}^{\infty}\left\langle u(x), \sum_{k=1}^{i} \beta_{i k} \psi_{k}(x)\right\rangle_{W_{2}^{2}} \bar{\psi}_{i}(x) \\
& =\sum_{i=1}^{\infty} \sum_{k=1}^{i} \beta_{i k}\left\langle u(x), \psi_{k}(x)\right\rangle_{W_{2}^{2}} \bar{\psi}_{i}(x)=\sum_{i=1}^{\infty} \sum_{k=1}^{i} \beta_{i k}\left\langle u(x), L^{*} \varphi_{k}(x)\right\rangle_{W_{2}^{2}} \bar{\psi}_{i}(x) \\
& =\sum_{i=1}^{\infty} \sum_{k=1}^{i} \beta_{i k}\left\langle L u(x), \varphi_{k}(x)\right\rangle_{W_{2}^{1}} \bar{\psi}_{i}(x)=\sum_{i=1}^{\infty} \sum_{k=1}^{i} \beta_{i k} L u\left(x_{k}\right) \bar{\psi}_{i}(x) .
\end{aligned}
$$

But since $u(x)$ is the exact solution of (3.1), then $L u(x)=F(x, u(x), T u(x), S u(x))$ and

$$
u(x)=\sum_{i=1}^{\infty} \sum_{k=1}^{i} \beta_{i k} F\left(x_{k}, u\left(x_{k}\right), T u\left(x_{k}\right), S u\left(x_{k}\right)\right) \bar{\psi}_{i}(x) .
$$

So, the proof of the theorem is complete.

Note that we denote to the approximate solution of $u(x)$ by

$$
u_{n}(x)=\sum_{i=1}^{n} \sum_{k=1}^{i} \beta_{i k} F\left(x_{k}, u\left(x_{k}\right), T u\left(x_{k}\right), S u\left(x_{k}\right)\right) \bar{\psi}_{i}(x)
$$

Theorem 3.3. If $u(x) \in W_{2}^{2}[a, b]$, then there exists $M>0$ such that $\left\|u^{(i)}(x)\right\|_{C} \leq M\|u(x)\|_{W_{2}^{2}}$, $i=0,1$, where $\|u(x)\|_{C}=\max _{a \leq x \leq b}|u(x)|$.

Proof. For any $x, y \in[a, b]$, we have $u^{(i)}(x)=\left\langle u(y), K_{x}^{(i)}(y)\right\rangle_{W_{2}^{2}}, i=0,1$. By the expression of $K_{x}(y)$, it follows that $\left\|K_{x}^{(i)}(y)\right\|_{W_{2}^{2}} \leq M_{i}, i=0,1$. Thus, $\left|u^{(i)}(x)\right|=\left|\left\langle u(x), K_{x}^{(i)}(x)\right\rangle_{W_{2}^{2}}\right| \leq$ $\|u(x)\|_{W_{2}^{2}}\left\|K_{x}^{(i)}(x)\right\|_{W_{2}^{2}} \leq M_{i}\|u(x)\|_{W_{2}^{2}}, i=0,1$. Hence, $\left\|u^{(i)}(x)\right\|_{C} \leq M\|u(x)\|_{W_{2}^{2}}, i=0,1$, where $M=\max \left\{M_{0}, M_{1}\right\}$. The proof is complete.

Corollary 3.4. The approximate solution $u_{n}(x)$ and its derivative $u_{n}^{\prime}(x)$ are uniformly convergent. 
Proof. By Theorems 3.2 and 3.3, for any $x \in[a, b]$, we get

$$
\begin{aligned}
& \left|u_{n}(x)-u(x)\right| \\
& \quad=\left|\left\langle u_{n}(x)-u(x), K_{x}(x)\right\rangle_{W_{2}^{2}}\right| \leq\left\|K_{x}(x)\right\|_{W_{2}^{2}}\left\|u_{n}(x)-u(x)\right\|_{W_{2}^{2}} \leq M_{0}\left\|u_{n}(x)-u(x)\right\|_{W_{2}^{2}} .
\end{aligned}
$$

On the other hand,

$$
\begin{aligned}
& \left|u_{n}^{\prime}(x)-u^{\prime}(x)\right| \\
& \quad=\left|\left\langle u_{n}(x)-u(x), K_{x}^{\prime}(x)\right\rangle_{W_{2}^{2}}\right| \leq\left\|K_{x}^{\prime}(x)\right\|_{W_{2}^{2}}\left\|u_{n}(x)-u(x)\right\|_{W_{2}^{2}} \leq M_{1}\left\|u_{n}(x)-u(x)\right\|_{W_{2}^{2}} .
\end{aligned}
$$

Hence, $\left|u_{n}^{(i)}(x)-u^{(i)}(x)\right| \leq\left\|u_{n}^{(i)}(x)-u^{(i)}(x)\right\|_{C} \leq M_{i}\left\|u_{n}(x)-u(x)\right\|_{W_{2}^{2}}$, where $M_{0}$ and $M_{1}$ are positive constants. Hence, if $\left\|u_{n}(x)-u(x)\right\|_{W_{2}^{2}} \rightarrow 0$ as $n \rightarrow \infty$, the approximate solutions $u_{n}(x)$ and $u_{n}^{\prime}(x)$ converge uniformly to the exact solution $u(x)$ and its derivative, respectively.

\section{Iterative Method and Convergence Theorem}

In this section, an iterative method of obtaining the solution of (3.1) is presented in the reproducing kernel space $W_{2}^{2}[a, b]$.

First of all, we will mention the following remark in order to solve (1.1) and (1.2) numerically. If (1.1) is linear, then the exact and approximate solutions can be obtained directly from (3.5) and (3.6), respectively. On the other hand, if (1.1) is nonlinear, then the exact and approximate solutions can be obtained using the following iterative method.

According to (3.5), the representation of the solution of (1.1) can be denoted by

$$
u(x)=\sum_{i=1}^{\infty} A_{i} \bar{\psi}_{i}(x)
$$

where $A_{i}=\sum_{k=1}^{i} \beta_{i k} F\left(x_{k}, u\left(x_{k}\right), T u\left(x_{k}\right), S u\left(x_{k}\right)\right)$. In fact, $A_{i}, i=1,2, \ldots$, in (4.1) are unknown, and we will approximate $A_{i}$ using known $B_{i}$. For a numerical computation, we define initial function $u_{0}\left(x_{1}\right)=0$ and the $n$-term approximation to $u(x)$ by

$$
u_{n}(x)=\sum_{i=1}^{n} B_{i} \bar{\psi}_{i}(x)
$$


where the coefficients $B_{i}$ are given as

$$
\begin{aligned}
& B_{1}=\beta_{11} F\left(x_{1}, u_{0}\left(x_{1}\right), T u_{0}\left(x_{1}\right), S u_{0}\left(x_{1}\right)\right), \\
& u_{1}(x)=B_{1} \bar{\psi}_{1}(x) \\
& B_{2}=\sum_{k=1}^{2} \beta_{2 k} F\left(x_{k}, u_{k-1}\left(x_{k}\right), T u_{k-1}\left(x_{k}\right), S u_{k-1}\left(x_{k}\right)\right), \\
& u_{2}(x)=\sum_{i=1}^{2} B_{i} \bar{\psi}_{i}(x) \\
& \vdots \\
& u_{n-1}(x)=\sum_{i=1}^{n-1} B_{i} \bar{\psi}_{i}(x), \\
& B_{n}=\sum_{k=1}^{n} \beta_{n k} F\left(x_{k}, u_{k-1}\left(x_{k}\right), T u_{k-1}\left(x_{k}\right), S u_{k-1}\left(x_{k}\right)\right) .
\end{aligned}
$$

We mention here the following remark: in the iterative process of (4.2), we can guarantee that the approximation $u_{n}(x)$ satisfies the initial condition (1.2).

Now, the approximate solution $u_{n}^{N}(x)$ can be obtained by taking finitely many terms in the series representation of $u_{n}(x)$ and

$$
u_{n}^{N}(x)=\sum_{i=1}^{N} \sum_{k=1}^{i} \beta_{i k} F\left(x_{k}, u_{n-1}\left(x_{k}\right), T u_{n-1}\left(x_{k}\right), S u_{n-1}\left(x_{k}\right)\right) \bar{\psi}_{i}(x) .
$$

Next, we will prove that $u_{n}(x)$ in the iterative formula (4.2) is convergent to the exact solution $u(x)$ of $(1.1)$.

Lemma 4.1. If $\left\|u_{n}(x)-u(x)\right\|_{W_{2}^{2}} \rightarrow 0, x_{n} \rightarrow y$ as $n \rightarrow \infty$ and $F(x, v, w, z)$ is continuous in $[a, b]$ with respect to $x, v, w, z$, for $x \in[a, b]$ and $v, w, z \in(-\infty, \infty)$, then as $n \rightarrow \infty$, one has $F\left(x_{n}, u_{n-1}\left(x_{n}\right), T u_{n-1}\left(x_{n}\right), S u_{n-1}\left(x_{n}\right)\right) \rightarrow F(y, u(y), T u(y), S u(y))$.

Proof. Firstly, we will prove that $u_{n-1}\left(x_{n}\right) \rightarrow u(y)$ in the sense of $\|\cdot\|_{W_{2}^{2}}$. Since

$$
\begin{aligned}
\left|u_{n-1}\left(x_{n}\right)-u(y)\right| & =\left|u_{n-1}\left(x_{n}\right)-u_{n-1}(y)+u_{n-1}(y)-u(y)\right| \\
& \leq\left|u_{n-1}\left(x_{n}\right)-u_{n-1}(y)\right|+\left|u_{n-1}(y)-u(y)\right|
\end{aligned}
$$

by reproducing kernel property of $K_{x}(y)$, we have $u_{n-1}\left(x_{n}\right)=\left\langle u_{n-1}(x), K_{x_{n}}(x)\right\rangle$ and $u_{n-1}(y)=$ $\left\langle u_{n-1}(x), K_{y}(x)\right\rangle$. Thus, $\left|u_{n-1}\left(x_{n}\right)-u_{n-1}(y)\right|=\left|\left\langle u_{n-1}(x), K_{x_{n}}(x)-K_{y}(x)\right\rangle_{W_{2}^{2}}\right| \leq\left\|u_{n-1}(x)\right\|_{W_{2}^{2}}$ $\left\|K_{x_{n}}(x)-K_{y}(x)\right\|_{W_{2}^{2}}$. From the symmetry of $K_{x}(y)$, it follows that $\left\|K_{x_{n}}(x)-K_{y}(x)\right\|_{W_{2}^{2}} \rightarrow 0$ as $n \rightarrow \infty$. Hence, $\left|u_{n-1}\left(x_{n}\right)-u_{n-1}(y)\right| \rightarrow 0$ as soon as $x_{n} \rightarrow y$.

On the other hand, by Corollary 3.4, for any $y \in[a, b]$, it holds that $\left|u_{n-1}(y)-u(y)\right| \rightarrow$ 0 . Therefore, $u_{n-1}\left(x_{n}\right) \rightarrow u(y)$ in the sense of $\|\cdot\|_{W_{2}^{2}}$ as $x_{n} \rightarrow y$ and $n \rightarrow \infty$. 
Thus, by means of the continuation of $G_{1}, G_{2}$, and $N$, it is obtained that $G_{1}\left(u_{n-1}\left(x_{n}\right)\right) \rightarrow$ $G_{1}(u(y)), G_{2}\left(u_{n-1}\left(x_{n}\right)\right) \rightarrow G_{2}(u(y))$, and $N\left(x_{n}, u_{n-1}\left(x_{n}\right)\right) \rightarrow N(y, u(y))$ as $n \rightarrow \infty$. This shows that $T u_{n-1}\left(x_{n}\right) \rightarrow T u(y)$ and $S u_{n-1}\left(x_{n}\right) \rightarrow S u(y)$ as $n \rightarrow \infty$. Hence, the continuity of $F$ gives the result.

Lemma 4.2. $\left\{u_{n}\right\}_{n=1}^{\infty}$ in (4.2) is monotonically increasing in the sense of the norm of $W_{2}^{2}[a, b]$.

Proof. By Theorem 3.1, $\left\{\bar{\psi}_{i}\right\}_{i=1}^{\infty}$ is the complete orthonormal system in the space $W_{2}^{2}[a, b]$. Hence, we have $\left\|u_{n}\right\|_{W_{2}^{2}}^{2}=\left\langle u_{n}(x), u_{n}(x)\right\rangle_{W_{2}^{2}}=\left\langle\sum_{i=1}^{n} B_{i} \bar{\psi}_{i}(x), \sum_{i=1}^{n} B_{i} \bar{\psi}_{i}(x)\right\rangle_{W_{2}^{2}}=\sum_{i=1}^{n}\left(B_{i}\right)^{2}$. Therefore, $\left\|u_{n}\right\|_{W_{2}^{2}}$ is monotonically increasing.

Lemma 4.3. One has $\operatorname{Lu}_{n}\left(x_{j}\right)=F\left(x_{j}, u_{j-1}\left(x_{j}\right), T u_{j-1}\left(x_{j}\right), S u_{j-1}\left(x_{j}\right)\right), j \leq n$.

Proof. The proof will be obtained by mathematical induction as follows: if $j \leq n$, then $L u_{n}\left(x_{j}\right)=\sum_{i=1}^{n} B_{i} L \bar{\psi}_{i}\left(x_{j}\right)=\sum_{i=1}^{n} B_{i}\left\langle L \bar{\psi}_{i}(x), \varphi_{j}(x)\right\rangle_{W_{2}^{1}}=\sum_{i=1}^{n} B_{i}\left\langle\bar{\psi}_{i}(x), L_{j}^{*} \varphi(x)\right\rangle_{W_{2}^{2}}=\sum_{i=1}^{n} B_{i}$ $\left\langle\bar{\psi}_{i}(x), \psi_{j}(x)\right\rangle_{W_{2}^{2}}$. Thus,

$$
L u_{n}\left(x_{j}\right)=\sum_{i=1}^{n} B_{i}\left\langle\bar{\psi}_{i}(x), \psi_{j}(x)\right\rangle_{W_{2}^{2}} .
$$

Multiplying both sides of (4.6) by $\beta_{j l}$, summing for $l$ from 1 to $j$, and using the orthogonality of $\left\{\bar{\psi}_{i}(x)\right\}_{i=1}^{\infty}$ yield that

$$
\begin{aligned}
\sum_{l=1}^{j} \beta_{j l} L u_{n}\left(x_{l}\right) & =\sum_{i=1}^{n} B_{i}\left\langle\bar{\psi}_{i}(x), \sum_{l=1}^{j} \beta_{j l} \psi_{l}(x)\right\rangle_{W_{2}^{2}}=\sum_{i=1}^{n} B_{i}\left\langle\bar{\psi}_{i}(x), \bar{\psi}_{j}(x)\right\rangle_{W_{2}^{2}}=B_{j} \\
& =\sum_{l=1}^{j} \beta_{j l} F\left(x_{l}, u_{l-1}\left(x_{l}\right), T u_{l-1}\left(x_{l}\right), S u_{l-1}\left(x_{l}\right)\right) .
\end{aligned}
$$

Now, if $j=1$, then $L u_{n}\left(x_{1}\right)=F\left(x_{1}, u_{0}\left(x_{1}\right), T u_{0}\left(x_{1}\right), S u_{0}\left(x_{1}\right)\right)$. On the other hand, if $j=2$, then $\beta_{21} L u_{n}\left(x_{1}\right)+\beta_{22} L u_{n}\left(x_{2}\right)=\beta_{21} F\left(x_{1}, u_{0}\left(x_{1}\right), T u_{0}\left(x_{1}\right), S u_{0}\left(x_{1}\right)\right)+\beta_{22} F\left(x_{2}, u_{1}\left(x_{2}\right)\right.$, $\left.T u_{1}\left(x_{2}\right), S u_{1}\left(x_{2}\right)\right)$. Thus, $\operatorname{Lu}_{n}\left(x_{2}\right)=F\left(x_{2}, u_{1}\left(x_{2}\right), T u_{1}\left(x_{2}\right), S u_{1}\left(x_{2}\right)\right)$. It is easy to see that $L u_{n}\left(x_{j}\right)=F\left(x_{j}, u_{j-1}\left(x_{j}\right), T u_{j-1}\left(x_{j}\right), S u_{j-1}\left(x_{j}\right)\right)$ by using mathematical induction.

Lemma 4.4. One has $\operatorname{Lu}_{n}\left(x_{j}\right)=\operatorname{Lu}\left(x_{j}\right), j \leq n$.

Proof. It is clear that on taking limits in (4.2) $u(x)=\sum_{i=1}^{\infty} B_{i} \bar{\psi}_{i}(x)$. Therefore, $u_{n}(x)=$ $P_{n} u(x)$, where $P_{n}$ is an orthogonal projector from $W_{2}^{2}[a, b]$ to Span $\left\{\psi_{1}, \psi_{2}, \ldots, \psi_{n}\right\}$. Thus, $L u_{n}\left(x_{j}\right)=\left\langle L u_{n}(x), \varphi_{j}(x)\right\rangle_{W_{2}^{1}}=\left\langle u_{n}(x), L_{j}^{*} \varphi(x)\right\rangle_{W_{2}^{2}}=\left\langle P_{n} u(x), \psi_{j}(x)\right\rangle_{W_{2}^{2}}=\left\langle u(x), P_{n} \psi_{j}(x)\right\rangle_{W_{2}^{2}}=$ $\left\langle u(x), \psi_{j}(x)\right\rangle_{W_{2}^{2}}=\left\langle L u(x), \varphi_{j}(x)\right\rangle_{W_{2}^{1}}=L u\left(x_{j}\right)$.

Theorem 4.5. Suppose that $\left\|u_{n}\right\|_{W_{2}^{2}}$ is bounded in (4.2). If $\left\{x_{i}\right\}_{i=1}^{\infty}$ is dense on $[a, b]$, then the $n$-term approximate solution $u_{n}(x)$ in the iterative formula (4.2) converges to the exact solution $u(x)$ of (3.1) in the space $W_{2}^{2}[a, b]$ and $u(x)=\sum_{i=1}^{\infty} B_{i} \bar{\psi}_{i}(x)$, where $B_{i}$ is given by (4.3). 
Proof. First of all, we will prove the convergence of $u_{n}(x)$. From (4.2), we infer that $u_{n+1}(x)=$ $u_{n}(x)+B_{n+1} \bar{\psi}_{n+1}(x)$. The orthogonality of $\left\{\bar{\psi}_{i}(x)\right\}_{i=1}^{\infty}$ yields that

$$
\left\|u_{n+1}\right\|_{W_{2}^{2}}^{2}=\left\|u_{n}\right\|_{W_{2}^{2}}^{2}+\left(B_{n+1}\right)^{2}=\left\|u_{n-1}\right\|_{W_{2}^{2}}^{2}+\left(B_{n}\right)^{2}+\left(B_{n+1}\right)^{2}=\cdots=\left\|u_{0}\right\|_{W_{2}^{2}}^{2}+\sum_{i=1}^{n+1}\left(B_{i}\right)^{2} .
$$

From Lemma 4.2, the sequence $\left\|u_{n}\right\|_{W_{2}^{2}}$ is monotonically increasing. Due to the condition that $\left\|u_{n}\right\|_{W_{2}^{2}}$ is bounded, $\left\|u_{n}\right\|_{W_{2}^{2}}$ is convergent as $n \rightarrow \infty$. Then, there exists a constant $c$ such that $\sum_{i=1}^{\infty}\left(B_{i}\right)^{2}=c$. This implies that $B_{i}=\sum_{k=1}^{i} \beta_{i k} F\left(x_{k}, u_{k-1}\left(x_{k}\right), T u_{k-1}\left(x_{k}\right), S u_{k-1}\left(x_{k}\right)\right) \in$ $l^{2}, i=1,2, \ldots$.

If $m>n$, using $\left(u_{m}-u_{m-1}\right) \perp\left(u_{m-1}-u_{m-2}\right) \perp \cdots \perp\left(u_{n+1}-u_{n}\right)$, then one gets

$$
\begin{aligned}
\left\|u_{m}(x)-u_{n}(x)\right\|_{W_{2}^{2}}^{2} & =\left\|u_{m}(x)-u_{m-1}(x)+u_{m-1}(x)-\cdots+u_{n+1}(x)-u_{n}(x)\right\|_{W_{2}^{2}}^{2} \\
& =\left\|u_{m}(x)-u_{m-1}(x)\right\|_{W_{2}^{2}}^{2}+\cdots+\left\|u_{n+1}(x)-u_{n}(x)\right\|_{W_{2}^{2}}^{2} .
\end{aligned}
$$

Furthermore, $\left\|u_{m}(x)-u_{m-1}(x)\right\|_{W_{2}^{2}}^{2}=\left(B_{m}\right)^{2}$. Consequently, $\left\|u_{m}(x)-u_{n}(x)\right\|_{W_{2}^{2}}^{2}=\sum_{i=n+1}^{m}$ $\left(B_{i}\right)^{2} \rightarrow 0$ as $n, m \rightarrow \infty$. Considering the completeness of the space $W_{2}^{2}[a, b]$, there exists a $u(x) \in W_{2}^{2}[a, b]$ such that $u_{n}(x) \rightarrow u(x)$ as $n \rightarrow \infty$ in the sense of $\|\cdot\|_{W_{2}^{2}}$.

Secondly, we will prove that $u(x)$ is the solution of (3.1). Since $\left\{x_{i}\right\}_{i=1}^{\infty}$ is dense on $[a, b]$, for any $x \in[a, b]$, there exists subsequence $\left\{x_{n_{j}}\right\}_{j=1}^{\infty}$ such that $x_{n_{j}} \rightarrow x$ as $j \rightarrow \infty$. From Lemmas 4.3 and 4.4 , it is easy to see that $L u\left(x_{n_{j}}\right)=F\left(x_{n_{j}}, u_{n_{j}-1}\left(x_{k}\right), T u_{n_{j}-1}\left(x_{k}\right), S u_{n_{j}-1}\left(x_{k}\right)\right)$. Hence, letting $j \rightarrow \infty$, by Lemma 4.1 and the continuity of $F$, we have $L u(x)=$ $F(x, u(x), T u(x), S u(x))$. That is, $u(x)$ is the solution of (3.1).

Since $\bar{\psi}_{i}(x) \in W_{2}^{2}[a, b]$, clearly, $u(x)$ satisfies the initial condition (1.2). In other words, $u(x)$ is the solution of (1.1) and (1.2), where $u(x)=\sum_{i=1}^{\infty} B_{i} \bar{\psi}_{i}(x)$ and $B_{i}$ is given by (4.3). The proof is complete.

Theorem 4.6. Assume that $u(x)$ is the solution of (3.1) and $r_{n}(x)$ is the difference between the approximate solution $u_{n}(x)$ and the exact solution $u(x)$. Then, $r_{n}(x)$ is monotonically decreasing in the sense of the norm of $W_{2}^{2}[a, b]$.

Proof. It obvious that $\left\|r_{n}(x)\right\|_{W_{2}^{2}}^{2}=\left\|u(x)-u_{n}(x)\right\|_{W_{2}^{2}}^{2}=\left\|\sum_{i=n+1}^{\infty} B_{i} \bar{\psi}_{i}(x)\right\|_{W_{2}^{2}}^{2}=\sum_{i=n+1}^{\infty}\left(B_{i}\right)^{2}$ and $\left\|r_{n-1}(x)\right\|_{W_{2}^{2}}^{2}=\sum_{i=n}^{\infty}\left(B_{i}\right)^{2}$. Thus, $\left\|r_{n}(x)\right\|_{W_{2}^{2}} \leq\left\|r_{n-1}(x)\right\|_{W_{2}^{2}}$; consequently, the difference $r_{n}(x)$ is monotonically decreasing in the sense of $\|\cdot\|_{W_{2}^{2}}$. So, the proof of the theorem is complete.

\section{Numerical Examples}

In this section, some numerical examples are studied to demonstrate the accuracy and applicability of the present method. Results obtained are compared with the exact solution of each example and are found to be in good agreement with each other. In the process of computation, all the symbolic and numerical computations were performed by using Mathematica 7.0 software package. 
Table 1: Numerical results for Example 5.1.

\begin{tabular}{lcccc}
\hline$x$ & Exact solution & Approximate solution & Absolute error & Relative error \\
\hline 0.16 & 0.0256 & 0.025600985200558 & $9.85201 \times 10^{-7}$ & $3.84844 \times 10^{-5}$ \\
0.32 & 0.1024 & 0.102401995676314 & $1.99568 \times 10^{-6}$ & $1.94890 \times 10^{-5}$ \\
0.48 & 0.2304 & 0.230403057350893 & $3.05735 \times 10^{-6}$ & $1.32698 \times 10^{-5}$ \\
0.64 & 0.4096 & 0.409604197461425 & $4.19746 \times 10^{-6}$ & $1.02477 \times 10^{-5}$ \\
0.80 & 0.6400 & 0.640005445257301 & $5.44526 \times 10^{-6}$ & $8.50821 \times 10^{-6}$ \\
0.96 & 0.9216 & 0.921606832750570 & $6.83275 \times 10^{-6}$ & $7.41401 \times 10^{-6}$ \\
\hline
\end{tabular}

Table 2: Numerical results of $u^{\prime}(x)$ for Example 5.1 .

\begin{tabular}{lcccc}
\hline$x$ & Exact solution & Approximate solution & Absolute error & Relative error \\
\hline 0.16 & 0.32 & 0.319999014799438 & $9.85201 \times 10^{-7}$ & $3.07875 \times 10^{-6}$ \\
0.32 & 0.64 & 0.639998004323680 & $1.99568 \times 10^{-6}$ & $3.11824 \times 10^{-6}$ \\
0.48 & 0.96 & 0.959996942649100 & $3.05735 \times 10^{-6}$ & $3.18474 \times 10^{-6}$ \\
0.64 & 1.28 & 1.279995802538568 & $4.19746 \times 10^{-6}$ & $3.27927 \times 10^{-6}$ \\
0.80 & 1.60 & 1.599994554742679 & $5.44526 \times 10^{-6}$ & $3.40329 \times 10^{-6}$ \\
0.96 & 1.92 & 1.919993167249420 & $6.83275 \times 10^{-6}$ & $3.55872 \times 10^{-6}$ \\
\hline
\end{tabular}

Example 5.1. Consider the nonlinear Fredholm-Volterra IDE:

$$
\begin{gathered}
u^{\prime}(x)+u(x)-\frac{1}{4} \int_{0}^{1} t u^{3}(t) d t+\frac{1}{2} \int_{0}^{x} x u^{2}(t) d t=g(x), \quad 0 \leq x, t \leq 1 \\
u(0)=0
\end{gathered}
$$

where $g(x)=(1 / 10) x^{6}+x^{2}+2 x-(1 / 32)$. The exact solution is $u(x)=x^{2}$.

Using RKHS method, taking $x_{i}=(i-1) /(N-1), i=1,2, \ldots, N$, with the reproducing kernel function $K_{x}(y)$ on $[0,1]$, the approximate solution $u_{n}^{N}(x)$ is calculated by $(4.4)$. The numerical results at some selected grid points for $N=26$ and $n=5$ are given in Table 1 .

As we mention, we used the grid nodes mentioned earlier in order to obtain approximate solutions. Moreover, it is possible to pick any point in $[a, b]$ and as well the approximate solution and its derivative will be applicable. Next, the numerical results for Example 5.1 at some selected gird nodes in $[0,1]$ of $u^{\prime}(x)$ are given in Table 2 .

Table 3 shows, a comparison between the absolute errors of our method together with triangular functions method [6], operational matrix with block-pulse functions method [7], and Hybrid Legendre polynomials and block-pulse functions method [8]. As it is evident from the comparison results, it was found that our method in comparison with the mentioned methods is better with a view to accuracy and utilization.

Example 5.2. Consider the nonlinear Fredholm-Volterra IDE:

$$
\begin{gathered}
u^{\prime}(x)+2 x u(x)-\int_{0}^{1}(x-t) u(t) d t-\int_{0}^{x}(x+t) u^{3}(t) d t=g(x), \quad 0 \leq x, t \leq 1, \\
u(0)=1,
\end{gathered}
$$


Table 3: Numerical comparison of absolute error for Example 5.1.

\begin{tabular}{lcccc}
\hline$x$ & Method in [6] & Method in [7] & Method in [8] & RKHS method \\
\hline 0.0 & 0 & 0 & 0 & 0 \\
0.1 & $1.6600 \times 10^{-4}$ & $2.1840 \times 10^{-3}$ & $3.1000 \times 10^{-5}$ & $6.1415 \times 10^{-7}$ \\
0.2 & $2.5400 \times 10^{-4}$ & $1.4630 \times 10^{-3}$ & $7.5000 \times 10^{-5}$ & $1.2345 \times 10^{-6}$ \\
0.3 & $2.6200 \times 10^{-4}$ & $1.6780 \times 10^{-3}$ & $1.7100 \times 10^{-4}$ & $1.8671 \times 10^{-6}$ \\
0.4 & $1.9100 \times 10^{-4}$ & $7.2390 \times 10^{-3}$ & $9.4000 \times 10^{-5}$ & $2.5185 \times 10^{-6}$ \\
0.5 & $4.1000 \times 10^{-5}$ & $1.6024 \times 10^{-2}$ & $2.2800 \times 10^{-4}$ & $3.1950 \times 10^{-6}$ \\
0.6 & $2.0200 \times 10^{-4}$ & $1.1480 \times 10^{-2}$ & $5.0200 \times 10^{-4}$ & $3.9035 \times 10^{-6}$ \\
0.7 & $2.8300 \times 10^{-4}$ & $4.5150 \times 10^{-3}$ & $5.8300 \times 10^{-4}$ & $4.6511 \times 10^{-6}$ \\
0.8 & $2.8300 \times 10^{-4}$ & $4.8720 \times 10^{-3}$ & $3.7400 \times 10^{-4}$ & $5.4453 \times 10^{-6}$ \\
0.9 & $2.0200 \times 10^{-4}$ & $1.6682 \times 10^{-2}$ & $4.7000 \times 10^{-5}$ & $6.2939 \times 10^{-6}$ \\
1.0 & $3.7000 \times 10^{-5}$ & $3.0914 \times 10^{-2}$ & $1.4000 \times 10^{-5}$ & $7.2055 \times 10^{-6}$ \\
\hline
\end{tabular}

Table 4: Numerical results for Example 5.2.

\begin{tabular}{lcccc}
\hline$x$ & Exact solution & Approximate solution & Absolute error & Relative error \\
\hline 0.0 & 1 & 1 & 0 & 0 \\
0.1 & 1.105170918075648 & 1.105171575587132 & $6.57511 \times 10^{-7}$ & $5.94941 \times 10^{-7}$ \\
0.2 & 1.221402758160170 & 1.221404273302208 & $1.51514 \times 10^{-6}$ & $1.24049 \times 10^{-6}$ \\
0.3 & 1.349858807576003 & 1.349861385895122 & $2.57832 \times 10^{-6}$ & $1.91007 \times 10^{-6}$ \\
0.4 & 1.491824697641270 & 1.491828549248914 & $3.85161 \times 10^{-6}$ & $2.58181 \times 10^{-6}$ \\
0.5 & 1.648721270700128 & 1.648726613238682 & $5.34254 \times 10^{-6}$ & $3.24041 \times 10^{-6}$ \\
0.6 & 1.822118800390509 & 1.822125866868782 & $7.06648 \times 10^{-6}$ & $3.87817 \times 10^{-6}$ \\
0.7 & 2.013752707470476 & 2.013761760093792 & $9.05262 \times 10^{-6}$ & $4.49540 \times 10^{-6}$ \\
0.8 & 2.225540928492467 & 2.225552279886237 & $1.13514 \times 10^{-5}$ & $5.10051 \times 10^{-6}$ \\
0.9 & 2.459603111156949 & 2.459617154977751 & $1.40438 \times 10^{-5}$ & $5.70979 \times 10^{-6}$ \\
1.0 & 2.718281828459045 & 2.718299082481196 & $1.72540 \times 10^{-5}$ & $6.34740 \times 10^{-6}$ \\
\hline
\end{tabular}

where $g(x)=(-(2 / 3) x+(1 / 9)) e^{3 x}+(2 x+1) e^{x}+((4 / 3)-e) x+(8 / 9)$. The exact solution is $u(x)=e^{x}$.

Using RKHS method, taking $x_{i}=(i-1) /(N-1), i=1,2, \ldots, N$, with the reproducing kernel function $K_{x}(y)$ on $[0,1]$, the approximate solution $u_{n}^{N}(x)$ is calculated by (4.4). The numerical results at some selected grid points for $N=26$ and $n=1$ are given in Table 4 .

The comparison among the RKHS solution besides the solutions of triangular functions [6], operational matrix with block-pulse functions solution [7], and exact solutions are shown in Table 5.

Example 5.3. Consider the nonlinear Fredholm-Volterra IDE:

$$
\begin{gathered}
u^{\prime}(x)+u^{2}(x)-\int_{0}^{1}(t+1) \sinh (u(t)-1) d t-\int_{0}^{x} x e^{u(t)} d t=g(x), \quad 0 \leq x, t \leq 1, \\
u(0)=1
\end{gathered}
$$


Table 5: Numerical comparison of approximate solution for Example 5.2.

\begin{tabular}{lcccc}
\hline$x$ & Exact solution & RKHS method & Method in [6] & Method in [7] \\
\hline 0.0 & 1.000000 & 1.000000 & 1.000000 & 1.000000 \\
0.1 & 1.105171 & 1.105172 & 1.105223 & 1.115708 \\
0.2 & 1.221403 & 1.221404 & 1.221494 & 1.225369 \\
0.3 & 1.349859 & 1.349861 & 1.349971 & 1.345821 \\
0.4 & 1.491825 & 1.491829 & 1.491933 & 1.478128 \\
0.5 & 1.648721 & 1.648727 & 1.648795 & 1.675019 \\
0.6 & 1.822119 & 1.822126 & 1.822484 & 1.839752 \\
0.7 & 2.013753 & 2.013762 & 2.014465 & 2.020740 \\
0.8 & 2.225541 & 2.225552 & 2.226719 & 2.219630 \\
0.9 & 2.459603 & 2.459617 & 2.461507 & 2.438278 \\
1.0 & 2.718280 & 2.718300 & 2.721505 & 2.678832 \\
\hline
\end{tabular}

Table 6: Numerical results for Example 5.3.

\begin{tabular}{lcccc}
\hline$x$ & Exact solution & Approximate solution & Absolute error & Relative error \\
\hline 0.16 & 1.148420005118273 & 1.148420410550930 & $4.05433 \times 10^{-7}$ & $3.53035 \times 10^{-7}$ \\
0.32 & 1.277631736598280 & 1.277632408078983 & $6.71481 \times 10^{-7}$ & $5.25567 \times 10^{-7}$ \\
0.48 & 1.392042087776024 & 1.392042943683538 & $8.55908 \times 10^{-7}$ & $6.14857 \times 10^{-7}$ \\
0.64 & 1.494696241836107 & 1.494697231133553 & $9.89297 \times 10^{-7}$ & $6.61872 \times 10^{-7}$ \\
0.80 & 1.587786664902119 & 1.587787753995242 & $1.08909 \times 10^{-6}$ & $6.85919 \times 10^{-7}$ \\
0.96 & 1.672944473242426 & 1.672945638649806 & $1.16541 \times 10^{-6}$ & $6.96620 \times 10^{-7}$ \\
\hline
\end{tabular}

where $g(x)=(\ln (x+1)+1)^{2}+(x+1)^{-1}-(e / 2)(x+2) x^{2}-(2 / 3)$. The exact solution is $u(x)=$ $1+\ln (x+1)$.

Using RKHS method, taking $x_{i}=(i-1) /(N-1), i=1,2, \ldots, N$, with the reproducing kernel function $K_{x}(y)$ on $[0,1]$, the approximate solution $u_{n}^{N}(x)$ is calculated by $(4.4)$. The numerical results at some selected grid points for $N=51$ and $n=1$ are given in Table 6 .

Example 5.4. Consider the nonlinear Fredholm-Volterra IDE:

$$
\begin{gathered}
u^{\prime}(x)+\cos (u(x))-\int_{0}^{1}(t+x) u(t) d t-\int_{0}^{x} t e^{u(t)} d t=g(x), \quad 0 \leq x, t \leq 1, \\
u(0)=1,
\end{gathered}
$$

where $g(x)=\cos (1-x)+e^{1-x}(1+x)-(1 / 2) x-(7 / 6)-e$. The exact solution is $u(x)=1-x$.

Using RKHS method, taking $x_{i}=(i-1) /(N-1), i=1,2, \ldots, N$, with the reproducing kernel function $K_{x}(y)$ on $[0,1]$, the approximate solution $u_{n}^{N}(x)$ is calculated by $(4.4)$. The numerical results at some selected grid points for $N=51$ and $n=5$ are given in Table 7 . 
Table 7: Numerical results for Example 5.4.

\begin{tabular}{lcccc}
\hline$x$ & Exact solution & Approximate solution & Absolute error & Relative error \\
\hline 0.16 & 0.84 & 0.840000573907808 & $5.73908 \times 10^{-7}$ & $6.83224 \times 10^{-7}$ \\
0.32 & 0.68 & 0.680001166402260 & $1.16640 \times 10^{-6}$ & $1.71530 \times 10^{-6}$ \\
0.48 & 0.52 & 0.520001763060855 & $1.76306 \times 10^{-6}$ & $3.39050 \times 10^{-6}$ \\
0.64 & 0.36 & 0.360002349461090 & $2.34946 \times 10^{-6}$ & $6.52628 \times 10^{-6}$ \\
0.80 & 0.20 & 0.200002911180463 & $2.91118 \times 10^{-6}$ & $1.45559 \times 10^{-5}$ \\
0.96 & 0.04 & 0.040003433796472 & $3.43380 \times 10^{-6}$ & $8.58449 \times 10^{-5}$ \\
\hline
\end{tabular}

Table 8: Numerical results for Example 5.5.

\begin{tabular}{lcccc}
\hline$x$ & Exact solution & Approximate solution & Absolute error & Relative error \\
\hline 0.16 & 0.16 & 0.159999994448885 & $5.55112 \times 10^{-9}$ & $3.46945 \times 10^{-8}$ \\
0.32 & 0.32 & 0.319999983346655 & $1.66533 \times 10^{-8}$ & $5.20417 \times 10^{-8}$ \\
0.48 & 0.48 & 0.479999983346655 & $1.66533 \times 10^{-8}$ & $3.46945 \times 10^{-8}$ \\
0.64 & 0.64 & 0.639999777955395 & $2.22045 \times 10^{-7}$ & $3.46945 \times 10^{-7}$ \\
0.80 & 0.80 & 0.799999555910790 & $4.44089 \times 10^{-7}$ & $5.55112 \times 10^{-7}$ \\
0.96 & 0.96 & 0.959999555910790 & $4.44089 \times 10^{-7}$ & $4.62593 \times 10^{-7}$ \\
\hline
\end{tabular}

Example 5.5. Consider the nonlinear Fredholm-Volterra IDE:

$$
\begin{gathered}
u^{\prime}(x)+x u^{2}(x)-\int_{0}^{1}(t+x)\left(1+u^{2}(t)\right) d t-\int_{0}^{x} x \cos (u(t)) d t=g(x), \quad 0 \leq x, t \leq 1, \\
u(0)=0,
\end{gathered}
$$

where $g(x)=-x \sin (x)+x^{3}-(4 / 3) x+(1 / 4)$. The exact solution is $u(x)=x$.

Using RKHS method, taking $x_{i}=(i-1) /(N-1), i=1,2, \ldots, N$ with the reproducing kernel function $K_{x}(y)$ on $[0,1]$, the approximate solution $u_{n}^{N}(x)$ is calculated by (4.4). The numerical results at some selected grid points for $N=26$ and $n=5$ are given in Table 8 .

\section{Conclusion}

In this paper, the RKHS method was employed to solve the nonlinear Fredholm-Volterra IDEs (1.1) and (1.2). The solution $u(x)$ and the approximate solution $u_{n}(x)$ are represented in the form of series in the space $W_{2}^{2}[a, b]$. Moreover, the approximate solution and its derivative converge uniformly to the exact solution and its derivative, respectively. Meanwhile, the error of the approximate solution is monotonically decreasing in the sense of the norm of $W_{2}^{2}[a, b]$.

\section{References}

[1] F. Bloom, "Asymptotic bounds for solutions to a system of damped integro-differential equations of electromagnetic theory," Journal of Mathematical Analysis and Applications, vol. 73, no. 2, pp. 524-542, 1980. 
[2] K. Holmåker, "Global asymptotic stability for a stationary solution of a system of integro-differential equations describing the formation of liver zones," SIAM Journal on Mathematical Analysis, vol. 24, no. 1, pp. 116-128, 1993.

[3] L. K. Forbes, S. Crozier, and D. M. Doddrell, "Calculating current densities and fields produced by shielded magnetic resonance imaging probes," SIAM Journal on Applied Mathematics, vol. 57, no. 2, pp. 401-425, 1997.

[4] R. P. Kanwal, Linear Integral Differential Equations Theory and Technique, Academic Press, New York, NY, USA, 1971.

[5] K. Maleknejad and Y. Mahmoudi, "Taylor polynomial solution of high-order nonlinear VolterraFredholm integro-differential equations," Applied Mathematics and Computation, vol. 145, no. 2-3, pp. 641-653, 2003.

[6] E. Babolian, Z. Masouri, and S. Hatamzadeh-Varmazyar, "Numerical solution of nonlinear VolterraFredholm integro-differential equations via direct method using triangular functions," Computers and Mathematics with Applications, vol. 58, no. 2, pp. 239-247, 2009.

[7] E. Babolian, Z. Masouri, and S. Hatamzadeh-Varmazyar, "New direct method to solve nonlinear Volterra-Fredholm integral and integro-differential equations using operational matrix with blockpulse functions," Progress In Electromagnetics Research B, vol. 8, pp. 59-76, 2008.

[8] K. Maleknejad, B. Basirat, and E. Hashemizadeh, "Hybrid Legendre polynomials and block-pulse functions approach for nonlinear Volterra-Fredholm integro-differential equations," Computers and Mathematics with Applications, vol. 61, no. 9, pp. 2821-2828, 2011.

[9] S. Momani and R. Qaralleh, "An efficient method for solving systems of fractional integro-differential equations," Computers and Mathematics with Applications, vol. 52, no. 3-4, pp. 459-470, 2006.

[10] M. Ghasemi, M. Tavassoli Kajani, and E. Babolian, "Application of He's homotopy perturbation method to nonlinear integro-differential equations," Applied Mathematics and Computation, vol. 188, no. 1, pp. 538-548, 2007.

[11] A. El-Ajou, O. Abu Arqub, and S. Momani, "Homotopy analysis method for second-order boundary value problems of integro-differential equations," Discrete Dynamics in Nature and Society. In press.

[12] A. Berlinet and C. Thomas-Agnan, Reproducing Kernel Hilbert Space in Probability and Statistics, Kluwer Academic, Boston, Mass, USA, 2004.

[13] M. Cui and Y. Lin, Nonlinear Numercial Analysis in the Reproducing Kernel Space, Nova Science, New York, NY, USA, 2008.

[14] A. Daniel, Reproducing Kernel Spaces and Applications, Springer, New York, NY, USA, 2003.

[15] M. Cui and H. Du, "Representation of exact solution for the nonlinear Volterra-Fredholm integral equations," Applied Mathematics and Computation, vol. 182, no. 2, pp. 1795-1802, 2006.

[16] H. Du and J. Shen, "Reproducing kernel method of solving singular integral equation with cosecant kernel," Journal of Mathematical Analysis and Applications, vol. 348, no. 1, pp. 308-314, 2008.

[17] F. Geng, "A new reproducing kernel Hilbert space method for solving nonlinear fourth-order boundary value problems," Applied Mathematics and Computation, vol. 213, no. 1, pp. 163-169, 2009.

[18] F. Geng, "Solving singular second order three-point boundary value problems using reproducing kernel Hilbert space method," Applied Mathematics and Computation, vol. 215, no. 6, pp. 2095-2102, 2009.

[19] F. Geng and M. Cui, "Solving singular nonlinear two-point boundary value problems in the reproducing kernel space," Journal of the Korean Mathematical Society, vol. 45, no. 3, pp. 631-644, 2008.

[20] F. Geng and M. Cui, "Solving a nonlinear system of second order boundary value problems," Journal of Mathematical Analysis and Applications, vol. 327, no. 2, pp. 1167-1181, 2007.

[21] F. Geng and M. Cui, "Solving singular nonlinear second-order periodic boundary value problems in the reproducing kernel space," Applied Mathematics and Computation, vol. 192, no. 2, pp. 389-398, 2007.

[22] J. Li, “A computational method for solving singularly perturbed two-point singular boundary value problem," International Journal of Mathematical Analysis, vol. 2, no. 21-24, pp. 1089-1096, 2008.

[23] C.-1. Li and M.-g. Cui, "The exact solution for solving a class nonlinear operator equations in the reproducing kernel space," Applied Mathematics and Computation, vol. 143, no. 2-3, pp. 393-399, 2003.

[24] Y. Li, F. Geng, and M. Cui, "The analytical solution of a system of nonlinear differential equations," International Journal of Mathematical Analysis, vol. 1, no. 9-12, pp. 451-462, 2007.

[25] Y. Z. Lin, M. G. Cui, and L. H. Yang, "Representation of the exact solution for a kind of nonlinear partial differential equation," Applied Mathematics Letters, vol. 19, no. 8, pp. 808-813, 2006.

[26] X. Lü and M. Cui, "Solving a singular system of two nonlinear ODEs," Applied Mathematics and Computation, vol. 198, no. 2, pp. 534-543, 2008. 
[27] L. Yang and M. Cui, "New algorithm for a class of nonlinear integro-differential equations in the reproducing kernel space," Applied Mathematics and Computation, vol. 174, no. 2, pp. 942-960, 2006.

[28] Y. Lin, P. Chung, and M. Cui, "A solution of an infinite system of quadratic equations in reproducing kernel space," Complex Analysis and Operator Theory, vol. 1, no. 4, pp. 571-579, 2007.

[29] M. Al-Smadi, O. Abu Arqub, and N. Shawagfeh, "Approximate solution of BVPs for 4th-order IDEs by using RKHS method," Applied Mathematical Sciences, vol. 6, pp. 2453-2464, 2012.

[30] Y. Zhou, M. Cui, and Y. Lin, "Numerical algorithm for parabolic problems with non-classical conditions," Journal of Computational and Applied Mathematics, vol. 230, no. 2, pp. 770-780, 2009. 


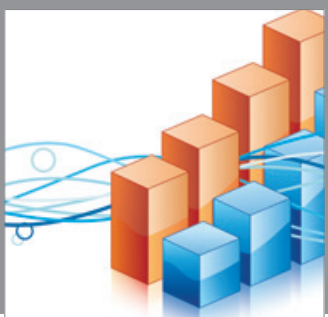

Advances in

Operations Research

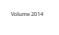

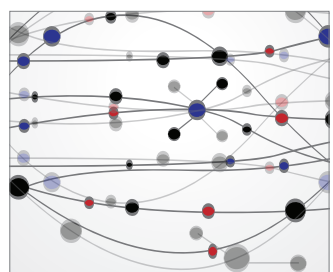

\section{The Scientific} World Journal
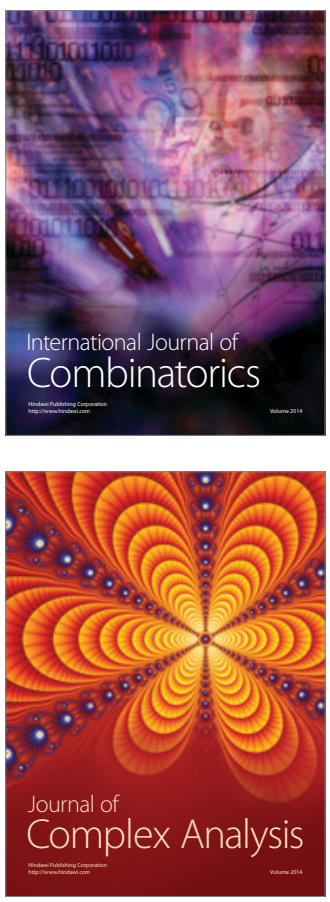

International Journal of

Mathematics and

Mathematical

Sciences
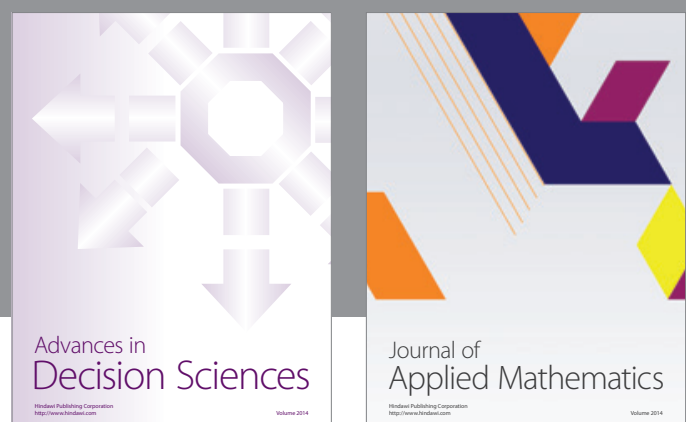

Journal of

Applied Mathematics
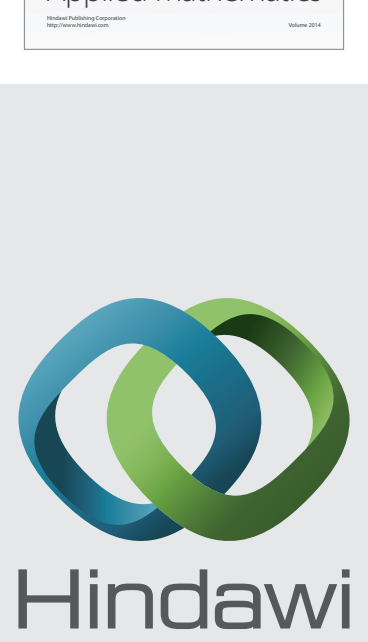

Submit your manuscripts at http://www.hindawi.com
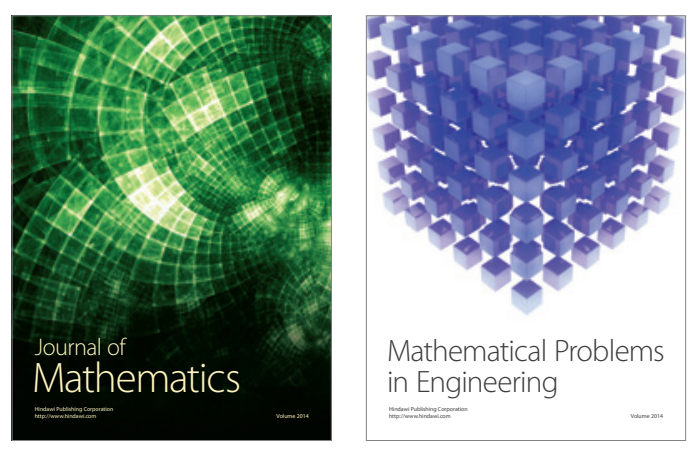

Mathematical Problems in Engineering
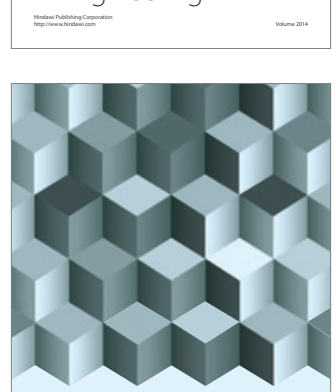

Journal of

Function Spaces
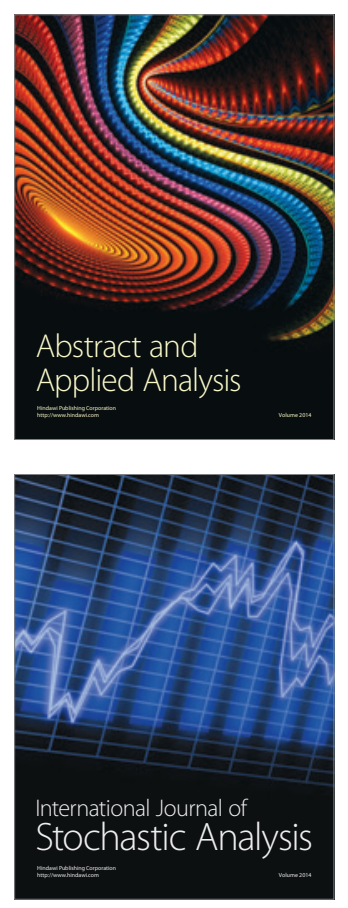

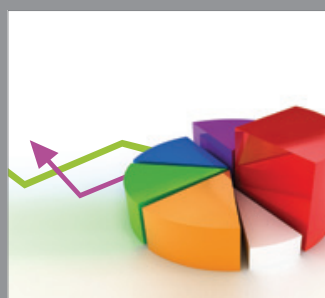

ournal of

Probability and Statistics

Promensencen
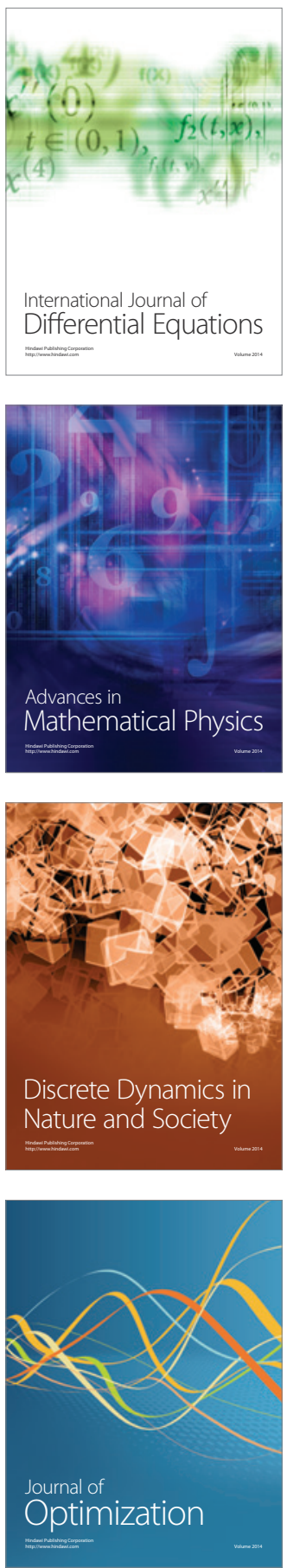